\title{
Phenytoin Sodium and Acetate-Maleate Buffered Balanced Salt Solutions are Physically Incompatible!
}

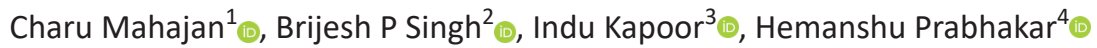 \\ Keywords: Balanced salt solutions, Incompatibility, Phenytoin. \\ Indian Journal of Critical Care Medicine (2021): 10.5005/jp-journals-10071-23756
}

Balanced salt solutions (BSS) are isotonic electrolyte solutions that help to maintain electrolyte balance in the body. Many newer compositions have come up in the last decade and are increasingly being used perioperatively. Phenytoin sodium is one of the commonest drugs used in neuroanesthesia practice, often used intraoperatively in high-risk head injury patients. We report our observation of the physical incompatibility of acetate-maleate buffered BSS and phenytoin sodium in our clinical practice.

A 44-years-old male sustained a severe traumatic brain injury and had an admission Glasgow coma scale (GCS) score of 5 . Non-contrast computed tomography head revealed right fronto-temporo-parietal acute subdural hematoma with multiple contusions. The patient was shifted to the operation theatre for hematoma evacuation and decompressive craniectomy. In addition to a $16-\mathrm{G}$ cannula inserted on the dorsum of the left hand, a central venous catheter was inserted in the right internal jugular vein. Normal saline $0.9 \%$ and acetate-maleate buffered BSS were used to replace the fluid deficits. Intraoperatively, the surgeon requested administration of a loading dose of phenytoin sodium ( $1 \mathrm{gm}$ ) that was diluted in $500 \mathrm{~mL}$ normal saline (NS). This was infused via another three-way connector in the already running infusion line in the dorsum of the hand. The drop rate was adjusted to run the drug very slowly. Soon after, on close observation, the formation of white precipitate was noticed in the tubing, distal to the three-way connector. The infusion was stopped immediately before the precipitated solution could enter circulation. On reviewing, BSS was noted to be flowing through the other end of the three-way connector that mixed with the NS-phenytoin causing precipitation. The BSS infusion was disconnected from the threeway and the tubing sets were changed. The drug was subsequently administered through an independent infusion line.

In neurosurgery, BSS maintain a more favorable metabolic profile and are often used intraoperatively in neurosurgical cases. ${ }^{1}$ However, those who are naïve in handling these relatively newer fluids, might not be aware of their interactions with other drugs. Various factors like $\mathrm{pH}$ reduction, effect of cosolvents, time delay from the dilution, temperature, and exposure to air may play a role in physical incompatibility. The $\mathrm{pH}$ of acetate-maleate buffered BSS is 5.1-5.9 while that of phenytoin sodium is 10-12. Propylene glycol, an organic co-solvent used to prepare phenytoin sodium injection interacts with glucose that results in enhancement of the precipitation of phenytoin. ${ }^{2}$ Hence, phenytoin sodium should not be diluted in dextrose solutions for infusion. In contrast, BSS containing calcium and acetate-maleate buffer may also possibly interact with propylene glycol to form a precipitate. This can be one of the several other reasons for physical incompatibility. In an experimental study
${ }^{1-4}$ Department of Neuroanaesthesiology and Critical Care, All India Institute of Medical Sciences (A.I.I.M.S.), New Delhi, Delhi, India

Corresponding Author: Charu Mahajan, Department of Neuroanaesthesiology and Critical Care, Neurosciences Centre, All India Institute of Medical Sciences (A.I.I.M.S.), New Delhi, Delhi, India, Phone: +91 7042132730, Fax:+91 1126588663, e-mail: charushrikul@gmail.com How to cite this article: Mahajan C, Singh BP, Kapoor I, Prabhakar H. Phenytoin Sodium and Acetate-Maleate Buffered Balanced Salt Solutions are Physically Incompatible! Indian J Crit Care Med 2021;25(3):351.

Source of support: Nil

Conflict of interest: None

done by Heiderich et al., authors found formation of a precipitate when phenytoin comes in contact with BSS and NS at various dilutions. ${ }^{3}$ For intravenous infusion, the standard fluid used for dilution is NS, and the concentration of phenytoin should not be more than $10 \mathrm{mg} / \mathrm{mL}$. At this concentration, precipitation of phenytoin with NS has never been reported in clinical practice.

We recommend administering phenytoin separately through a completely independent intravenous line to avoid accidental admixture with other intravenous fluids especially while using three-way connectors.

\section{OrCID}

Charu Mahajan 강t.//orcid.org/0000-0003-3712-9281

Brijesh P Singh @ https://orcid.org/0000-0003-1220-9381

Indu Kapoor (1) https://orcid.org/0000-0002-3065-4940

Hemanshu Prabhakar @ https://orcid.org/0000-0001-7830-3296

\section{References}

1. Hafizah M, Liu CY, Ooi JS. Normal saline versus balanced-salt solution as intravenous fluid therapy during neurosurgery: effects on acidbase balance and electrolytes. J Neurosurg Sci 2017;61(3):263-270. DOI: 10.23736/S0390-5616.16.03221-5.

2. Onuki Y, Ikegami-Kawai M, Ishitsuka K, Hyashi Y, Takayama K. A 5\% glucose infusion fluid provokes significant precipitation of phenytoin sodium injection via interruption of the cosolvent effect of propylene glycol. Chem Pharm Bull (Tokyo) 2012;60(1):86-93. DOI: 10.1248/ cpb.60.86.

3. Heiderich S, Jürgens J, Rudolf D, Dennhardt N, Echtermeyer F, Leffler A, et al. Compatibility of common drugs with acetate-containing balanced electrolyte solutions in pediatric anesthesia. Paediatr Anaesth 2016;26(6):590-598. DOI: 10.1111/pan.12889. 